\title{
An overview on how cumulus cells interact with the oocyte in a condition with elevated NEFA levels in dairy cows
}

\author{
Hilde Aardema*, Helena T.A. van Tol, Peter L.A.M. Vos \\ Department of Farm Animal Health, Faculty of Veterinary Medicine, Utrecht University, Yalelaan 7, 3584 CL Utrecht, the Netherlands
}

\section{A R T I C L E I N F O}

\section{Keywords:}

Cumulus

Oocyte

NEFA

Lipid

Fertility

Cow

\begin{abstract}
A B S T R A C T
Metabolic stress in humans and animals is associated with impaired fertility. A major characteristic of metabolic stress is elevated levels of free fatty acids (NEFAs) in blood due to mobilization of body fat reserves. Dairy cows undergo a period of metabolic stress during the pericalving period, the so-called negative energy balance (NEB) in the early weeks postpartum. At the time of NEB, both saturated and unsaturated NEFAs are mobilized to serve as an alternative energy supply for cells, however in particular saturated NEFAs can have a detrimental effect on somatic cells. Circulating NEFAs are also reflected in the follicular fluid of ovarian follicles and hence reach the cumulus-oocyte-complex (COC), which implies a potential risk for the developing oocyte. To this end, the current review focusses on the impact of NEFAs on the quality of the oocyte.
\end{abstract}

\section{Introduction}

Metabolic stress conditions, like obesity in humans and the negative energy balance in cows, have been associated with reduced fertility. One of the major characteristics of metabolic stress are elevated levels of free fatty acids (NEFA) in the circulation, and the levels of NEFA from blood are reflected in the follicular fluid (Leroy et al., 2005; Jungheim et al., 2011; Yang et al., 2012; Aardema et al., 2013; Valckx et al., 2014a,b; Aardema et al., 2015). Several groups investigated the impact of elevated levels of NEFA on the oocyte and whether this may explain the observed reduced fertility during metabolic stress. There appears to be a huge difference in the effect on the oocyte depending on the type of NEFA to which the oocyte is exposed. The most abundant saturated NEFAs in follicular fluid, palmitic and stearic acid, appear to have a dose-dependent negative impact on the competence of the oocyte to develop into an embryo (Leroy et al., 2005; Wu et al., 2010; Aardema et al., 2011). In contrast to the impact of mono-unsaturated oleic acid, the most abundant unsaturated NEFA in follicular fluid, that appears to be non-toxic until a high level for the maturing oocyte and is able to prevent a lipotoxic impact of saturated NEFA (Aardema et al., 2011). Interestingly, levels of mono-unsaturated oleic acid are relatively high in follicular fluid also during metabolic stress (Leroy et al., 2005; Aardema et al., 2013, 2015). The cumulus cells around the oocyte appear to protect the oocyte against elevated levels of NEFA by conversion of the potentially toxic saturated into mono-unsaturated NEFA and save storage of the fatty acids in lipid droplets (Aardema et al., 2017). It is intriguing how the mature follicle appears to be capable to affectively protect the oocyte against short term exposure to elevated NEFA levels, by the relatively high level of oleic acid in the follicular fluid and the protective role of cumulus cells. The current review will describe the latest findings on how the cumulus-oocyte-complex responds on his NEFA environment.

\footnotetext{
* Corresponding author.

E-mail address: h.aardema@uu.nl (H. Aardema).
} 


\section{Free fatty acids}

In adipose tissue the main lipid reservoir of the body is stored. The lipid in adipocytes is stored as triacylglycerol (TAG; 3 fatty acids esterified to glycerol) in lipid droplets. The energetic value of fatty acid is high, the complete aerobic catabolism of 1 mol of stearic acid versus $3 \mathrm{~mol}$ of glucose (for comparing in both cases $18 \mathrm{C}$ atoms) yields 5-6 x more energy in the form of ATP. During periods of metabolic stress, like for example obesity, but also energy scarcity during a negative energy balance, fatty acids are mobilized from body fat reserves and complexed to albumin for transportation via blood to supply the different tissues in the body that take up fatty acids from blood, which results in elevated levels of NEFAs in blood. Elevated levels of NEFAs in the circulation are also reflected in the follicular fluid in both humans and cows, nevertheless the NEFA concentration and composition between blood and follicular fluid differs (Leroy et al., 2005; Jungheim et al., 2011; Yang et al., 2012; Aardema et al., 2013; Valckx et al., 2014a, b; Aardema et al., 2015). Many distinct NEFAs present in the circulation, one way to classify these different types of NEFA is by the absence or presence of double bonds between carbon atoms. Saturated NEFAs lack a double bond, unsaturated NEFAs carry one (mono-unsaturated) or more double bonds (poly-unsaturated) in their carbon skeleton that changes their structure from rigid (saturated) into a more flexible (unsaturated) structure with a reduced melting temperature. Several studies describe a lipotoxic impact of saturated palmitic and stearic acid, the most abundant saturated NEFAs in the circulation, on different somatic cell types (Mu et al., 2001; Listenberger et al., 2003; Coll et al., 2008). In contrast, mono-unsaturated oleic acid, the most abundant mono-unsaturated NEFAs in the circulation, has in general a moderate impact on somatic cells (Listenberger et al., 2003; Coll et al., 2008).

To approach the oocyte, NEFAs need to pass the blood-follicle barrier. The blood-follicle barrier is formed by theca cells, the basal membrane and granulosa cells; passage depends on both size (70-300 kDa) and charge of the metabolite (Shalgi et al., 1973; Cran et al., 1976; Gosden et al., 1988; Fortune, 1994; Jaspard et al., 1997; Hess et al., 1998). Proteins pass the blood-follicle barrier easier when positively charged, nevertheless small proteins, for example albumin ( $66 \mathrm{kDa})$, can freely pass the barrier independent of the negative charge and NEFAs complexed to albumin are present in the follicular fluid. The permeability of negatively charged substances is increased in response to the ovulatory surge (Hess et al., 1998). One class of lipoproteins, the high density lipoproteins (HDLs), are in contrast to low density and very low density lipoproteins, able to pass the blood-follicle-barrier. HDLs are rich in cholesteryl-esters (cholesterol with an esterified fatty acid) and contain low amounts of TAG. Follicular fluid contains approximately a total concentration of 1.8-2.0 mM fatty acids, which is mostly present in lipoproteins (cholesteryl-ester; $0.7 \mathrm{mM}$ and fatty acids of the phospholipid layer 1.1 mM) (Jaspard et al., 1997; Valckx et al., 2014a, b). During normal physiological conditions approximately $10 \%$ of the fatty acids present in follicular fluid is complexed to albumin as NEFA (0.23 mM). During metabolic stress conditions the total concentrations of HDL in both blood and follicular fluid appears not to be affected (Valckx et al., 2012; Aardema et al., 2013); whether the fatty acid composition of HDL is affected is to our knowledge not known. In contrast, NEFA levels massively increase in both blood and follicular fluid during metabolic stress (Leroy et al., 2005; Aardema et al., 2013). However, there is a difference in the NEFA composition between blood and follicular fluid. One of the most remarkable differences is the relatively high concentration of mono-unsaturated oleic acid in follicular fluid (Leroy et al., 2005; Aardema et al., 2013, 2015). The distinct NEFA composition between blood and follicular fluid may result from a selective transfer of fatty acids or metabolization and/ or storage in the cells that form the blood-follicle barrier. At this point, there is still a lot unknown about the potentially selective transfer of fatty acids from blood towards the follicular fluid. Hopefully future research can shed a light on the function of the blood-follicle barrier in relation to the transfer of NEFAs.

\section{Cumulus cells regulate fatty acid transfer to the oocyte}

After passage of the blood-follicle barrier, the cumulus cell layer around the oocyte forms the second natural 'barrier' between blood and the oocyte. Before NEFAs present in the follicular fluid can reach the oocyte, passage via cumulus cells is unavoidable. The expanded cumulus cell layer of, in particular, in vivo maturing oocytes forms a molecular filter that allows only a restricted transport of small metabolites to the oocyte (Dunning et al., 2012; Dumesic et al., 2015; Russell et al., 2016; Del Collado et al., 2017). The lack of a functional cumulus cell layer around the oocyte indeed results in a significantly higher lipid load in the oocyte (Lolicato et al., 2015). Furthermore, after removing cumulus cells at $8 \mathrm{~h}$ of maturation, exposure to levels of saturated stearic acid results in a significant drop in oocyte developmental competence in contrast to exposed oocytes from intact COCs or to non-exposed denuded oocytes (Aardema et al., 2017). Cumulus cells express CD36, the enzyme involved in the extracellular uptake of fatty acids by somatic cells (Uzbekova et al., 2015). The gap junctions that form a tight connection between the cumulus cells and the oocyte contain fatty acid binding proteins, specific proteins that transport fatty acids indicating that cumulus cells actively coordinate the transfer of fatty acids to the oocyte (Uzbekova et al., 2015; Del Collado et al., 2017). Blockage of these transzonal projections with cytochalasin B results in a reduction of the lipid amount present in the oocyte (Del Collado et al., 2017). Although, the reduction in lipid amount can also be explained by increased lipid degradation due to the presence of cytochalasin $\mathrm{B}$, which was not verified in this paper, this observation is a first indication for an active exchange of fatty acids between cumulus cells and the oocyte. Nevertheless, the fatty acid exchange between cumulus cells and the oocyte still is a topic that certainly needs further investigation. A few intriguing questions are; how and until what level interact the oocyte and cumulus cells in the exchange of fatty acids, does fatty acid transport occur in one or both directions and is there a selection in the fatty acids that are transported to the oocyte. Hopefully more aspects of this intriguing area of research will be elucidated in the coming years. 


\section{The oocyte 'senses' its lipid environment}

The lipid composition of oocytes appears to depend on the metabolic environment of the oocyte. Oocytes contain numerous lipid droplets that are coated with PAT proteins, perilipin-2 (formerly named ADRP) and perilipin-3 (formerly named TIP47), for support and stabilization of the lipid droplet (Aardema et al., 2011; Xu et al., 2018). Oocytes seem to be capable to take up fatty acids from their environment and have the inner cellular machinery for de novo lipid metabolism (Cetica et al., 2002; Auclair et al., 2013; Uzbekova et al., 2015). CD36, important for the uptake of long chain fatty acids, is likewise in cumulus cells also expressed in oocytes. In liver cells, it has been suggested that CD36 acts as a protective metabolic sensor during conditions of lipid overload (Garbacz et al., 2016). The expression of CD36 increases in response to elevated glucose and insulin levels (Uzbekova et al., 2015; Wilson et al., 2016; Garbacz et al., 2016; Ly et al., 2017). Likely the storage of exogenous fatty acids by the oocyte, in the presence of elevated NEFA levels in follicular fluid, may thus depend on glucose and consequently on insulin levels. As a consequence, one may argue that the response of the oocyte to elevated levels of NEFA may largely depend on the energetic condition of the dam. Physiologically energy rich conditions, i.e. high levels in glucose and consequently insulin, drive cells to store energy. Indeed, mouse oocytes exposed to lipid rich follicular fluid of obese women during IVM show an increased amount of intracellular lipid; likewise oocytes retrieved from obese mice demonstrate a higher amount of lipid compared to controls (Wu et al., 2010; Yang et al., 2012). The exposure to elevated levels of NEFAs during in vitro maturation, in a glucose rich medium, also results in rapid incorporation of fatty acids in lipid droplets in bovine oocytes (Aardema et al., 2011; Lolicato et al., 2015). In contrast, elevated levels of NEFA in bovine follicular fluid during a period of negative energy balance do not affect the lipid content of in vivo derived bovine oocytes (Aardema et al., 2013). Although in first instance the response of the oocyte to exogenous FFA may seem unpredictable, when the energetic condition is taken into account and hence the level of glucose to which the oocyte is exposed, the response appears to be in line with the physiologic response. This observation is definitely a research area that deserves attention in future experiments. It also indicates that it is important to not solely focus on the effect of fatty acids, but to also consider the energetic condition in relation to the impact of fatty acids on the oocyte. However, the present biological variation in oocytes from the same animal, in animals of the same species and between distinct species, has to be taken into account as well as this may also result in a distinct response of individual oocytes in comparable metabolic conditions (Sturmey et al., 2009).

Apart from taking the energetic condition and biological variation into account, it is also important to consider the time period during which COCs are exposed to NEFA. Final maturation in cows takes around 22-24 $\mathrm{h}$ in the cow and this is also the time for in vitro maturation during conventional IVP. Consequently, in most studies COCs are exposed to elevated NEFA concentrations during a relatively short period of time compared to the in vivo time length of exposure of the oocyte to elevated levels of NEFA during metabolic stress. One of the first attempts, as far as we know, to investigate the impact of NEFA on the follicle and its enclosed oocyte during an extended period of time is performed by Valckx et al. (Valckx et al., 2014a, b). In this study murine studies follicles were exposed to different NEFA conditions from the preantral stage onwards during 13 continuous days of culture. Exposure to conditions of elevated levels of NEFA, either high levels of stearic acid, oleic acid or a mixture of NEFA, reduced the blastocyst and hatching rate in the case of oleic acid, compared to the condition with a low basal level of NEFA (Valckx et al., 2014a, b). Whereas, there was only a moderate effect of oleic acid on follicular growth. The in this study observed negative effect of long term exposure of the oocyte in the follicle to oleic acid, is in sharp contrast to the moderate impact of exposure to high levels of oleic acid on the oocyte (Leroy et al., 2005; Aardema et al., 2011). The distinct impact of oleic acid may partly be explained by the different species used for the studies, murine versus bovine, or distinct media conditions like the inclusion of FBS. Nevertheless this study demonstrates that there is a need for models that closely mimic the in vivo situation to improve our understanding of the impact of NEFA on the oocyte.

Interestingly, seasonal changes can also affect the fatty acid composition of the oocyte. Zeron et al., demonstrated that the lipid composition of membranes of oocytes from Israeli Holstein cows shows seasonal variation containing a significantly higher level of unsaturated fatty acids in winter compared to oocyte membranes in summer (Zeron et al., 2001). The increased amount of unsaturated fatty acids during the winter period caused a $\Delta \mathrm{T}$ of $6{ }^{\circ} \mathrm{C}$ in the melting temperature of membranes between summer and winter analysed oocytes (Zeron et al., 2001). Unsaturated fatty acids lower the melting temperature of membranes, a phenomenon in somatic cells to compensate for a reduced temperature, and to maintain membrane plasticity (Schumann, 2016). However an intriguing question is how the shift in the fatty acid composition in oocytes is realized. On the one hand, a distinct diet during the summer and winter time may affect the supply of fatty acids and potentially the composition of the membranes. However, for example additional grazing, resulting in a net higher intake of unsaturated fatty acids, is not applicable to the Israeli situation as cows are kept indoors throughout the year. It remains intriguing how the fatty acid composition in the membrane is regulated, and whether; 1) oocytes are self-regulatory in adapting the membrane composition of fatty acids or 2) another driver, like cumulus cells, is involved in this process and 3) the basis for the fatty acid switch in membranes is depending on a food change or the outer temperature. Certainly more studies are needed to unravel this phenomenon and to generate a better understanding about the underlying mechanisms of fatty acid changes in the oocyte.

\section{Maturing oocytes exposed to elevated NEFA levels remain developmentally competent}

Oocytes derived from COCs that are exposed to saturated NEFA concentrations during final maturation have a, dose-dependent, reduced competence to develop into a blastocyst (Aardema et al., 2011). Exposure to saturated NEFA levels results in increased apoptosis in granulosa and cumulus cells (Mu et al., 2001; Leroy et al., 2005). The loss of functional cells may challenge the barrier function of the cumulus cells and hence enlarge the impact of saturated NEFA on the oocyte. Interestingly, the dramatic impact of saturated NEFA is counteracted by the simultaneous exposure to mono-unsaturated oleic acid (Aardema et al., 2011). The balancing 
presence of oleic acid in combination with elevated levels of saturated NEFA may explain the non-affected developmental competence of oocytes exposed to elevated levels of NEFA in vivo (Aardema et al., 2013). In particular, because the levels of oleic acid appear to be relatively high in follicular fluid (Leroy et al., 2005; Aardema et al., 2013, 2015). The contrast in the response of the oocyte on saturated and mono-unsaturated NEFA has also been observed for other cell types. In this respect, the distinct impact of mono-unsaturated fatty acid versus saturated on cells has been attributed to a different intracellular distribution of the fatty acids. Mono-unsaturated fatty acids like oleic and palmitoleic acid are easily stored and are primarily distributed towards lipid droplets, whereas, saturated fatty acids alone are directed to apoptotic pathways in somatic cell types while in combination with monounsaturated fatty acids storage in lipid droplets is more likely (Listenberger et al., 2003; Coll et al., 2008; Henique et al., 2010). Cumulus cells indeed massively accumulate lipid when the relatively oleic acid rich NEFA levels in the follicular fluid are increased, while the lipid composition in the oocyte remains unaffected (Aardema et al., 2013). Recent research showed that alpha-linolenic also seems capable to prevent a lipotoxic impact by saturated NEFA on the oocyte. However, in contrast to oleic acid which, even until a dose of $500 \mu \mathrm{M}$, showed no negative impact on the oocyte, a concentration of $50 \mu \mathrm{M}$ alpha-linolenic is already toxic for the oocyte (W. F. Marei et al., 2009; Aardema et al., 2011; W. F. A. Marei et al., 2017). All these findings point to a remarkably potent effect of oleic acid in counteracting the negative impact of saturated NEFA on the oocyte and a broad area in which oleic acid concentrations are safe for the oocyte.

\section{Cumulus cells protect the oocyte against exposure to excessive NEFA levels}

The lipid storage of excessive fatty acid levels in follicular fluid by cumulus cells during metabolic stress serves as a protection mechanism for maturing oocytes (Aardema et al., 2013). Lipid storage in response to the exposure to mono-unsaturated fatty acids has been observed in several somatic cell types and appears to be the main cause that mono-unsaturated NEFA counteracts the potentially negative impact of saturated NEFA on cells, as these fatty acids are redirected from the apoptotic pathway and safely stored in lipid droplets together with mono-unsaturated NEFA. Indeed, when COCs were exposed to elevated levels of NEFA, the increased storage of fatty acids was dominated by oleic acid (Aardema et al., 2013). Another previously described option to prevent the negative impact of fatty acids in the cell is to direct fatty acids towards $\beta$-oxidation in mitochondria. For example, in skeletal muscle an increase of $\beta$-oxidation activity was demonstrated in response to oleic acid (Henique et al., 2010). Hereby, carnitinepalmitoyl-transferase-I (CPT-I) is the rate limiting enzyme for $\beta$-oxidation and it is involved in the transport of fatty acids into the matrix of the mitochondria. Interestingly, cumulus cells do express CPT-I at relatively high levels (Dunning et al., 2010; Sanchez-Lazo et al., 2014; Uzbekova et al., 2015), which may protect the oocyte against potentially toxic NEFA, but the concept whether increased $\beta$-oxidation in COCs in the presence of elevated levels of NEFA protects the oocyte has not been tested, as far as we know. However, our group showed that elevated levels of in particular saturated NEFA appear to increase the level of ROS in the oocyte (Lolicato et al., 2015). An interesting recent review proposes that a balance in pyruvate and fatty acid oxidation is crucial in oocytes and contributes to the maintenance of a desired low ROS level in the oocyte (Bradley and Swann, 2019). To this end, this is certainly an important field for future research.

Recently an alternative option for protection of the oocyte by cumulus cells has been elucidated, desaturation of potentially toxic saturated fatty acids into mono-unsaturated fatty acid (Aardema et al., 2017). The key enzyme responsible for the conversion of saturated stearic acid into mono-unsaturated oleic acid, by the formation of a double carbon bond at the $\Delta 9$ position of saturated fatty acid, is stearoyl-CoA desaturase (SCD). In cumulus cells both mRNA and protein are expressed, while SCD was not detectable in oocytes (Aardema et al., 2017). SCD activity in cumulus cells appears to be of crucial importance to protect the oocyte against the exposure to fatty acid stress. Specific inhibition of the SCD enzyme in the presence of saturated stearic acid reduced the developmental competence of the oocyte to a similar extent, as exposure of denuded oocytes to saturated stearic acid (Aardema et al., 2017). This finding indicates that SCD activity in cumulus cells and hence the consequent conversion of potentially toxic saturated fatty acid into mono-unsaturated fatty acid, with an in general moderate impact on cells, is a prominent factor in the protection mechanism of the oocyte against fatty acid stress (see also Fig. 1). Furthermore, SCD activity in cumulus cells in the presence of stearic acid resulted in a significant reduction in the rate of apoptosis in the cumulus cells and hence increased lipid droplet storage of fatty acids dominated by oleic acid (Aardema et al., 2017). Cumulus cells thus appear to be of fundamental importance for the protection of the oocyte in a lipid rich environment.

The composition of mono-unsaturated free fatty acids in follicular fluid may be related to SCD activity in granulosa and cumulus cells, and needs further investigation. It appears that SCD expression, and thus the potency of desaturation of fatty acids, is also strongly related to the fatty acid composition and in particular the presence of mono-unsaturated fatty acids in milk (Garnsworthy et al., 2010; Bouwman et al., 2011; Rincon et al., 2012). The hypothetical situation that SCD expression and the free fatty acid composition of follicular fluid are related is intriguing and may have important implications for the resilience of the dairy cow to a NEB and hence on the fertility performance of individual cows. A relationship between SCD and fertility is of particular interest because it has been documented that the SCD activity varies among individual cows (Soyeurt et al., 2008; Garnsworthy et al., 2010).

\section{The consequence of a NEB on fertility in practice}

When the timing of insemination is considered in line with the preceding information, one may realize that under normal conditions cows will not be inseminated during the period of NEB. In general cows are inseminated at the earliest around 50-60 days postpartum i.e. after the severest period of metabolic stress. This timing implies that the peri-ovulatory follicle and its oocyte are not exposed to elevated levels of circulating NEFA at the final stage of their development, while most research so far focuses on the 


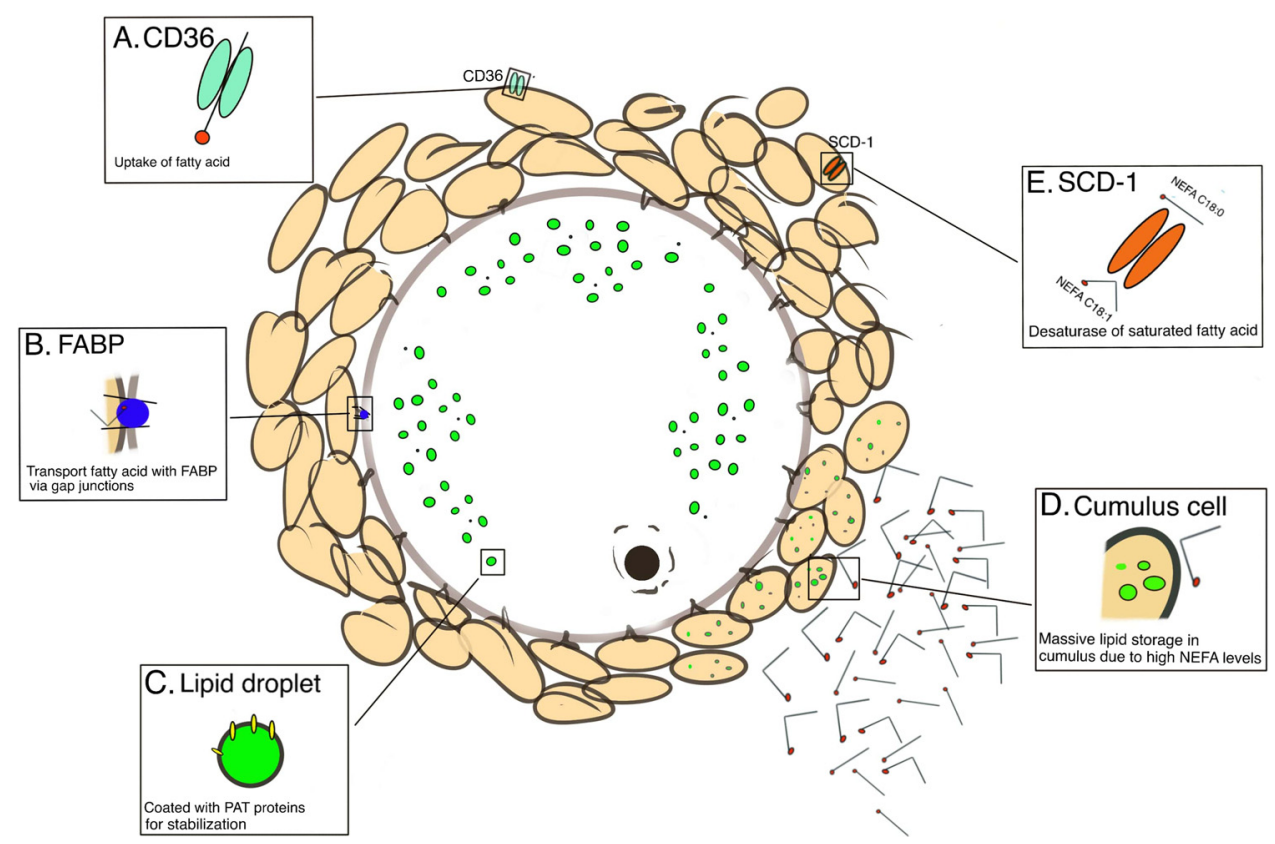

Fig. 1. The response of the cumulus-oocyte-complex to fatty acids.

External fatty acid from the follicular fluid can be taken up by cumulus cells via CD36 (A). The exchange of fatty acids between cumulus cells and the oocyte appears to occur via gap junctional transport with fatty acid binding proteins (FABP, B). In the oocyte fatty acids can be stored in lipid droplets, coated by PAT proteins (C). Elevated levels of NEFA in follicular fluid result in a massive storage of fatty acids in the lipid droplets of cumulus cells (D), and together with the conversion of potentially toxic saturated fatty acids in harmless mono-unsaturated fatty acids by SCD-1 activity in cumulus cells (E) and protects the oocyte against lipotoxicity.

impact of NEFA during this final maturation stage. Furthermore, at the final stage of maturation, oocytes seem to be fairly well protected against elevated NEFA levels by the oleic acid enriched follicular fluid and the protection by cumulus cells (Aardema et al., 2011, 2013; Aardema et al., 2017). To investigate the potential impact of metabolic stress during the early postpartum period on the fertility of the cow we need to focus on a different period during follicular development, being the early stages of follicular growth and development. The entire growth phase of the follicle, i.e. from the recruitment of the primordial follicle until the pre-ovulatory follicular stage, takes around 90-100 days in the cow (Britt, 1992). This indicates that the oocyte candidate to be fertilized, at the due insemination period of the cow post-partum, was exposed to the NEB months before and as a consequence developed during a phase of elevated levels of NEFA in the early weeks post-partum. At the start of follicular development, in the early follicular growth phase, the follicle contains only one single layer of granulosa cells surrounding the oocyte, and the follicle gradually develops into a structure that consists of several cell layers of theca, granulosa and cumulus cells during subsequent growth stages (Fair et al., 1997). One may speculate that around the secondary follicular stage, at which stage follicles start to become more metabolically active, follicles may be in particular prone to environmental changes and to metabolic stress (Fair et al., 1997). The previously shown necessity of a vital cumulus cell layer for protection of the oocyte triggers the question whether oocytes are protected against NEFA when cumulus cells are lacking during the early stages of (preantral) follicular development (Aardema et al., 2013, 2017). Counting backwards from the time of insemination of the cow, for example at day 70 post-partum, this indicates that these oocytes were fully exposed to the NEB related metabolic stress effects during the early stages of follicle development where the buffering follicular fluid and protecting cumulus cells were not yet present. Exposure of those early oocytes in pre-antral follicles to toxic reagents like elevated levels of NEFA may result in oocytes of inferior quality at the time of fertilization, however it is extremely challenging to test this hypothesis with experimental models. Nevertheless, Walters et al. (Walters et al., 2002) demonstrated that around 70 days postpartum the quality of bovine oocytes derived from antral follicles that started their development during the NEB period, was reduced. In an in vitro murine model, as mentioned earlier, it has been shown that NEFA exposure of follicles from the preantral stage onwards, during 13 continuous days, results in oocytes with a reduced competence to develop into a blastocyst (Valckx et al., 2014a, b). These studies provide evidence for the well-known Britt hypothesis, which states that the observed reduced fertility in cows experiencing a NEB may result from a carryover effect via the oocytes exposed to metabolic stress during an early stage of follicular development (Britt, 1992). However, an Irish study in which dairy and beef heifers were exposed to either energy restriction, to mimic the NEB, or a control diet for 50 days preceding AI demonstrated a positive carry over effect of feed restriction on the pregnancy rates (Parr et al., 2015). This study challenges the Britt hypothesis, as in this study the NEB and the induced metabolic changes and elevated FFA levels were not related with a reduced fertility. Hopefully the potential effects of the NEB at an early stage of follicular development is elucidated further in the near future. An indirect approach to investigate the proposed impact of a NEB status on oocyte growth and hence quality during the early stages of follicular development may be via manipulations during the transition period of the cow, for example by means of diets that are highly enriched with 'encapsulated fatty acids', fatty acids packed in for example calcium salts to 
overcome the biohydrogenation from unsaturated to saturated fatty acids in the rumen of the cow (Jenkins and Palmquist, 1984; Nafikov and Beitz, 2007). It has been shown that fatty acids from the diet are reflected in the total fatty acid fraction, including the fatty acids present in lipoproteins, of blood and follicular fluid (Wonnacott et al., 2010; Zachut et al., 2011). Many studies have focused on the effects of feeding commercially available conjugated poly-unsaturated fatty acids, like linoleic (C18:2) and linolenic acid (C18:3), with different aims including the improvement of fertility, with variable outcomes (Santos et al., 2008; Leroy et al., 2014; Rodney et al., 2015, 2018). Ideally, future studies will investigate the effect of (dietary) fatty acid interventions on oocyte quality during specifically the early stages of follicular development to generate better insight on the impact of metabolic changes during the NEB on oocytes at early stages of development.

\section{Concluding remarks}

Metabolic stress during the period of NEB in dairy cows, characterized by elevated levels of circulating NEFA, in the early postpartum period has been related with hampered fertility in cows. There appears to be a major difference in the impact of either saturated, potentially toxic, and unsaturated NEFA on the developmental competence of the oocyte. Furthermore, during the final stage of maturation both the relatively high oleic acid enriched follicular fluid and the protective mechanism of the cumulus cells appear to protect the oocyte effectively against the undesired fatty acid stress. An enigma in literature exists on the potential effect of elevated levels of NEFA on oocytes during earlier stages of follicular development. Whereas, the presumed candidate oocytes to be inseminated in the near future are fully exposed to the NEB metabolic effects during their early start of their development, at a time when protection by follicular fluid and cumulus cells is lacking. Future studies should focus on the potential impact of elevated levels of circulating NEFA during the early stages of follicular development and elucidate more precise the proposed impact on fertility in dairy cows under practical conditions.

\section{Competing interests}

The authors declare no competing interests.

\section{References}

Aardema, H., Gadella, B.M., van de Lest, C.H., Brouwers, J.F., Stout, T.A., Roelen, B.A., Vos, P.L., 2015. Free fatty acid levels in fluid of dominant follicles at the preferred insemination time in dairy cows are not affected by early postpartum fatty acid stress. J. Dairy Sci. 98, 2322-2336.

Aardema, H., Lolicato, F., van de Lest, C.H., Brouwers, J.F., Vaandrager, A.B., van Tol, H.T., Roelen, B.A., Vos, P.L., Helms, J.B., Gadella, B.M., 2013. Bovine cumulus cells protect maturing oocytes from increased fatty acid levels by massive intracellular lipid storage. Biol. Reprod. 88, 164.

Aardema, H., van Tol, H.T.A., Wubbolts, R.W., Brouwers, J.F.H.M., Gadella, B.M., Roelen, B.A.J., 2017. Stearoyl-CoA desaturase activity in bovine cumulus cells protects the oocyte against saturated fatty acid stress. Biol. Reprod. 96, 982-992.

Aardema, H., Vos, P.L., Lolicato, F., Roelen, B.A., Knijn, H.M., Vaandrager, A.B., Helms, J.B., Gadella, B.M., 2011. Oleic acid prevents detrimental effects of saturated fatty acids on bovine oocyte developmental competence. Biol. Reprod. 85, 62-69.

Auclair, S., Uzbekov, R., Elis, S., Sanchez, L., Kireev, I., Lardic, L., Dalbies-Tran, R., Uzbekova, S., 2013. Absence of cumulus cells during in vitro maturation affects lipid metabolism in bovine oocytes. Am. J. Physiol. Endocrinol. Metab. 304, E599-613.

Bouwman, A.C., Bovenhuis, H., Visker, M.H., van Arendonk, J.A., 2011. Genome-wide association of milk fatty acids in dutch dairy cattle. BMC Genet. 12 43-2156$12-43$.

Bradley, J., Swann, K., 2019. Mitochondria and lipid metabolism in mammalian oocytes and early embryos. Int. J. Dev. Biol. 63, $93-103$.

Britt, J.H., 1992. Impacts of early postpartum metabolism on follicular development and fertility. Bov. Proc. 24, 39-43.

Cetica, P., Pintos, L., Dalvit, G., Beconi, M., 2002. Activity of key enzymes involved in glucose and triglyceride catabolism during bovine oocyte maturation in vitro. Reproduction. 124, 675-681.

Coll, T., Eyre, E., Rodriguez-Calvo, R., Palomer, X., Sanchez, R.M., Merlos, M., Laguna, J.C., Vazquez-Carrera, M., 2008. Oleate reverses palmitate-induced insulin resistance and inflammation in skeletal muscle cells. J. Biol. Chem. 283, 11107-11116.

Cran, D.G., Moor, R.M., Hay, M.F., 1976. Permeability of ovarian follicles to electron-dense macromolecules. Acta Endocrinol. (Copenh). 82, 631-636.

Del Collado, M., da Silveira, J.C., Sangalli, J.R., Andrade, G.M., Sousa, L.R.D.S., Silva, L.A., Meirelles, F.V., Perecin, F., 2017. Fatty acid binding protein 3 and transzonal projections are involved in lipid accumulation during in vitro maturation of bovine oocytes. Sci. Rep. 7 2645-017-02467-9.

Dumesic, D.A., Meldrum, D.R., Katz-Jaffe, M.G., Krisher, R.L., Schoolcraft, W.B., 2015. Oocyte environment: follicular fluid and cumulus cells are critical for oocyte health. Fertil. Steril. 103, 303-316.

Dunning, K.R., Cashman, K., Russell, D.L., Thompson, J.G., Norman, R.J., Robker, R.L., 2010. Beta-oxidation is essential for mouse oocyte developmental competence and early embryo development. Biol. Reprod.

Dunning, K.R., Watson, L.N., Sharkey, D.J., Brown, H.M., Norman, R.J., Thompson, J.G., Robker, R.L., Russell, D.L., 2012. Molecular filtration properties of the mouse expanded cumulus matrix: controlled supply of metabolites and extracellular signals to cumulus cells and the oocyte. Biol. Reprod. 87, 89.

Fair, T., Hulshof, S.C., Hyttel, P., Greve, T., Boland, M., 1997. Oocyte ultrastructure in bovine primordial to early tertiary follicles. Anat. Embryol. (Berl). 195, $327-336$.

Fortune, J.E., 1994. Ovarian follicular growth and development in mammals. Biol. Reprod. 50, $225-232$.

Garbacz, W.G., Lu, P., Miller, T.M., Poloyac, S.M., Eyre, N.S., Mayrhofer, G., Xu, M., Ren, S., Xie, W., 2016. Hepatic overexpression of CD36 improves glycogen homeostasis and attenuates high-fat diet-induced hepatic steatosis and insulin resistance. Mol. Cell. Biol. 36, $2715-2727$.

Garnsworthy, P.C., Feng, S., Lock, A.L., Royal, M.D., 2010. Short communication: Heritability of milk fatty acid composition and stearoyl-CoA desaturase indices in dairy cows. J. Dairy Sci. 93, 1743-1748.

Gosden, R.G., Hunter, R.H., Telfer, E., Torrance, C., Brown, N., 1988. Physiological factors underlying the formation of ovarian follicular fluid. J. Reprod. Fertil. 82, $813-825$.

Henique, C., Mansouri, A., Fumey, G., Lenoir, V., Girard, J., Bouillaud, F., Prip-Buus, C., Cohen, I., 2010. Increased mitochondrial fatty acid oxidation is sufficient to protect skeletal muscle cells from palmitate-induced apoptosis. J. Biol. Chem. 285, 36818-36827.

Hess, K.A., Chen, L., Larsen, W.J., 1998. The ovarian blood follicle barrier is both charge- and size-selective in mice. Biol. Reprod. 58, 705-711.

Jaspard, B., Fournier, N., Vieitez, G., Atger, V., Barbaras, R., Vieu, C., Manent, J., Chap, H., Perret, B., Collet, X., 1997. Structural and functional comparison of HDL from homologous human plasma and follicular fluid. A model for extravascular fluid. Arterioscler. Thromb. Vasc. Biol. 17, 1605-1613.

Jenkins, T.C., Palmquist, D.L., 1984. Effect of fatty acids or calcium soaps on rumen and total nutrient digestibility of dairy rations. J. Dairy Sci. 67, 978-986.

Jungheim, E.S., Macones, G.A., Odem, R.R., Patterson, B.W., Lanzendorf, S.E., Ratts, V.S., Moley, K.H., 2011. Associations between free fatty acids, cumulus oocyte 
complex morphology and ovarian function during in vitro fertilization. Fertil. Steril. 95, 1970-1974.

Leroy, J.L., Sturmey, R.G., Van Hoeck, V., De Bie, J., McKeegan, P.J., Bols, P.E., 2014. Dietary fat supplementation and the consequences for oocyte and embryo quality: Hype or significant benefit for dairy cow reproduction? Reprod. Domest. Anim. 49, 353-361.

Leroy, J.L., Vanholder, T., Mateusen, B., Christophe, A., Opsomer, G., de Kruif, A., Genicot, G., Van Soom, A., 2005. Non-esterified fatty acids in follicular fluid of dairy cows and their effect on developmental capacity of bovine oocytes in vitro. Reproduction. 130, 485-495.

Listenberger, L.L., Han, X., Lewis, S.E., Cases, S., Farese Jr, R.V., Ory, D.S., Schaffer, J.E., 2003. Triglyceride accumulation protects against fatty acid-induced lipotoxicity. Proc. Natl. Acad. Sci. U. S. A. 100, 3077-3082.

Lolicato, F., Brouwers, J.F., de Lest, C.H., Wubbolts, R., Aardema, H., Priore, P., Roelen, B.A., Helms, J.B., Gadella, B.M., 2015. The cumulus cell layer protects the bovine maturing oocyte against fatty acid-induced lipotoxicity. Biol. Reprod. 92, 16.

Ly, L.D., Xu, S., Choi, S.K., Ha, C.M., Thoudam, T., Cha, S.K., Wiederkehr, A., Wollheim, C.B., Lee, I.K., Park, K.S., 2017. Oxidative stress and calcium dysregulation by palmitate in type 2 diabetes. Exp. Mol. Med. 49, e291.

Marei, W.F., Wathes, D.C., Fouladi-Nashta, A.A., 2009. The effect of linolenic acid on bovine oocyte maturation and development. Biol. Reprod. 81, 1064-1072.

Marei, W.F.A., De Bie, J., Mohey-Elsaeed, O., Wydooghe, E., Bols, P.E.J., Leroy, J.L.M.R., 2017. Alpha-linolenic acid protects the developmental capacity of bovine cumulus-oocyte complexes matured under lipotoxic conditions in vitro. Biol. Reprod. 96, 1181-1196.

Mu, Y.M., Yanase, T., Nishi, Y., Tanaka, A., Saito, M., Jin, C.H., Mukasa, C., Okabe, T., Nomura, M., Goto, K., Nawata, H., 2001. Saturated FFAs, palmitic acid and stearic acid, induce apoptosis in human granulosa cells. Endocrinology. 142, 3590-3597.

Nafikov, R.A., Beitz, D.C., 2007. Carbohydrate and lipid metabolism in farm animals. J. Nutr. 137, $702-705$.

Parr, M.H., Crowe, M.A., Lonergan, P., Evans, A.C., Fair, T., Diskin, M.G., 2015. The concurrent and carry over effects of long term changes in energy intake before insemination on pregnancy per artificial insemination in heifers. Anim. Reprod. Sci. 157, 87-94.

Rincon, G., Islas-Trejo, A., Castillo, A.R., Bauman, D.E., German, B.J., Medrano, J.F., 2012. Polymorphisms in genes in the SREBP1 signalling pathway and SCD are associated with milk fatty acid composition in holstein cattle. J. Dairy Res. 79, 66-75.

Rodney, R.M., Celi, P., Scott, W., Breinhild, K., Lean, I.J., 2015. Effects of dietary fat on fertility of dairy cattle: a meta-analysis and meta-regression. J. Dairy Sci. 98, 5601-5620.

Rodney, R.M., Celi, P., Scott, W., Breinhild, K., Santos, J.E.P., Lean, I.J., 2018. Effects of nutrition on the fertility of lactating dairy cattle. J. Dairy Sci. 101, 5115-5133.

Russell, D.L., Gilchrist, R.B., Brown, H.M., Thompson, J.G., 2016. Bidirectional communication between cumulus cells and the oocyte: Old hands and new players? Theriogenology 86, 62-68.

Sanchez-Lazo, L., Brisard, D., Elis, S., Maillard, V., Uzbekov, R., Labas, V., Desmarchais, A., Papillier, P., Monget, P., Uzbekova, S., 2014. Fatty acid synthesis and oxidation in cumulus cells support oocyte maturation in bovine. Mol. Endocrinol. 28, 1502-1521.

Santos, J.E., Bilby, T.R., Thatcher, W.W., Staples, C.R., Silvestre, F.T., 2008. Long chain fatty acids of diet as factors influencing reproduction in cattle. Reprod. Domest. Anim. 43 (Suppl 2), 23-30.

Schumann, J., 2016. It is all about fluidity: fatty acids and macrophage phagocytosis. Eur. J. Pharmacol. 785, 18-23.

Shalgi, R., Kraicer, P., Rimon, A., Pinto, M., Soferman, N., 1973. Proteins of human follicular fluid: the blood-follicle barrier. Fertil. Steril. $24,429-434$.

Soyeurt, H., Dehareng, F., Mayeres, P., Bertozzi, C., Gengler, N., 2008. Variation of delta 9-desaturase activity in dairy cattle. J. Dairy Sci. 91, 3211-3224.

Sturmey, R.G., Reis, A., Leese, H.J., McEvoy, T.G., 2009. Role of fatty acids in energy provision during oocyte maturation and early embryo development. Reprod. Domest. Anim. 44 (Suppl 3), 50-58.

Uzbekova, S., Elis, S., Teixeira-Gomes, A.P., Desmarchais, A., Maillard, V., Labas, V., 2015. MALDI mass spectrometry imaging of lipids and gene expression reveals differences in fatty acid metabolism between follicular compartments in porcine ovaries. Biology (Basel). 4, 216-236.

Valckx, S.D., Arias-Alvarez, M., De Pauw, I., Fievez, V., Vlaeminck, B., Fransen, E., Bols, P.E., Leroy, J.L., 2014a. Fatty acid composition of the follicular fluid of normal weight, overweight and obese women undergoing assisted reproductive treatment: a descriptive cross-sectional study. Reprod. Biol. Endocrinol. 12 13-782712-13.

Valckx, S.D., De Pauw, I., De Neubourg, D., Inion, I., Berth, M., Fransen, E., Bols, P.E., Leroy, J.L., 2012. BMI-related metabolic composition of the follicular fluid of women undergoing assisted reproductive treatment and the consequences for oocyte and embryo quality. Hum. Reprod. 27, $3531-3539$.

Valckx, S.D., Van Hoeck, V., Arias-Alvarez, M., Maillo, V., Lopez-Cardona, A.P., Gutierrez-Adan, A., Berth, M., Cortvrindt, R., Bols, P.E., Leroy, J.L., 2014b. Elevated non-esterified fatty acid concentrations during in vitro murine follicle growth alter follicular physiology and reduce oocyte developmental competence. Fertil. Steril. 102 1769-76.e1.

Walters, A.H., Bailey, T.L., Pearson, R.E., Gwazdauskas, F.C., 2002. Parity-related changes in bovine follicle and oocyte populations, oocyte quality, and hormones to 90 days postpartum. J. Dairy Sci. 85, 824-832.

Wilson, C.G., Tran, J.L., Erion, D.M., Vera, N.B., Febbraio, M., Weiss, E.J., 2016. Hepatocyte-specific disruption of CD36 attenuates fatty liver and improves insulin sensitivity in HFD-fed mice. Endocrinology 157, 570-585.

Wonnacott, K.E., Kwong, W.Y., Hughes, J., Salter, A.M., Lea, R.G., Garnsworthy, P.C., Sinclair, K.D., 2010. Dietary omega-3 and -6 polyunsaturated fatty acids affect the composition and development of sheep granulosa cells, oocytes and embryos. Reproduction 139, 57-69.

Wu, L.L., Dunning, K.R., Yang, X., Russell, D.L., Lane, M., Norman, R.J., Robker, R.L., 2010. High-fat diet causes lipotoxicity responses in cumulus-oocyte complexes and decreased fertilization rates. Endocrinology 151, 5438-5445.

Xu, M., Zeng, Y., Chi, D., Si, L., Qu, X., Li, J., 2018. The dynamic pattern of PLIN3 in pig oocytes and cumulus cells during in vitro maturation. Zygote 26, 40-49.

Yang, X., Wu, L.L., Chura, L.R., Liang, X., Lane, M., Norman, R.J., Robker, R.L., 2012. Exposure to lipid-rich follicular fluid is associated with endoplasmic reticulum stress and impaired oocyte maturation in cumulus-oocyte complexes. Fertil. Steril. 97 1438-1435.

Zachut, M., Arieli, A., Moallem, U., 2011. Incorporation of dietary n-3 fatty acids into ovarian compartments in dairy cows and the effects on hormonal and behavioral patterns around estrus. Reproduction 141, 833-840.

Zeron, Y., Ocheretny, A., Kedar, O., Borochov, A., Sklan, D., Arav, A., 2001. Seasonal changes in bovine fertility: Relation to developmental competence of oocytes, membrane properties and fatty acid composition of follicles. Reproduction 121, 447-454. 